\title{
CHEMICAL \\ PHYSICS LETTERS
}

Volume 44, No. 1, 15 November 1976

VIBRONIC RELAXATION IN THE $S_{1}$ STATE OF RHODAMINE DYE SOLUTIONS

A. PENZKOFER, W. FALKENSTEIN and W. KAISER

Physik Department der Technischen Universität Mn̈chen,

Munich, Germany

pp. $82-87$

NORTH-HOLLAND PUBLISHING COMPANY - AMSTERDAM 


\title{
VIBRONIC RELAXATION IN THE $S_{1}$ STATE OF RHODAMINE DYE SOLUTIONS
}

\author{
A. PENZKOFER, W. FALKENSTEIN and W. KAISER \\ Physik Department der Technischen Universitåt Müchen, \\ Munich, Germany
}

Received 2 August 1976

\begin{abstract}
The relaxation of vibronic energy levels was investigated by two different techniques. (A) The transmission of picosecond pulses was measured as a function of the incident peak intensity and $(B)$ the transmission of a second probe pulse was studied versus delay time. Population lifetimes of the excited levels between $0.5 \mathrm{ps}$ and 1.0 ps were found for rhodamine $6 \mathrm{G}$ and rhodamine $\mathrm{B}$ in the solvents water, ethanol, ethylene glycol and glycerol. The lifetimes are independent of solvent viscosity.
\end{abstract}

The relaxation of excited rhodamine molecules in the $S_{1}$ state has been studied by numerous authors [1-7]. Several papers [1-3] were concerned with the delayed amplification of a probe pulse after excitation by an intense pump pulse. Other investigations [4-6] measured the delayed appearance of Stokes shifted spontaneous fluorescence. In all these experiments the emitting and the corresponding final states are not known. The emitting vibrational levels vary during and immediately after the excitation pulse until the molecules settle into the relatively long-lived lowest $S_{1}$ state.

In this letter we report on measurements which determine the population lifetime $\tau$ of vibrational energy states located several hundred wave numbers above the bottom of the first excited singlet state $S_{1}$. Two different experimental techniques were used to determine the same relaxation time $\tau$.

(A) The energy transmission $T_{\mathrm{L}}$ of a single intense picosecond laser pulse was measured as a function of input peak intensity $I_{0 \mathrm{~L}}$. The nonlinear transmission of the molecules is compared with model calculations to provide the desired time constant $\tau$. Very rapid relaxation of the excited states leads to less effective bleaching at a fixed input intensity. We note that for relaxation times $\tau$ longer than the pulse duration $t_{\mathrm{p}}$ only one half of the molecules can be excited while for relaxation times $\tau$ shorter than $t_{\mathrm{p}}$ all molecules can be promoted to excited energy states.

(B) After the excitation by the first strong light pulse, the energy transmission $T_{\|}$of a weak interrogating pulse was measured as a function of delay time $t_{\mathrm{D}}$. The probe pulse had the same frequency, pulse duration, polarization and spatial distribution as the pump pulse. This probe pulse monitors the transient population of the initially excited vibrational states. The probe transmission is equal to the pump transmission when pump and probe beam coincide $\left(t_{\mathrm{D}}=0\right)$. At a delay time of $t_{\mathrm{D}} \approx t_{\mathrm{p}} / 2$ the probe transmission is largest. The transmission of the delayed probe pulse decreases while the molecules decay from the optically excited energy states to intermediate levels. If the relaxation time $\tau$ is considerably shorter than the exciting pulse duration $t_{\mathrm{p}}$, the molecules relax during the excitation pulse and the transmission of the probe pulse remains constant with increasing delay.

Our experimental set-up is depicted schematically in fig. 1. A mode-locked Nd-glass laser system was used in our investigations [8]. Single pulses were selected from the beginning of the pulse trains with an electrooptical shutter and passed subsequently through a Ndglass amplifier. The amplified single picosecond light pulses were band width limited with a duration of approximately $5 \mathrm{ps}$ (fwhm), a band width of $\Delta \tilde{\nu} \approx 3 \mathrm{~cm}^{-1}$ (fwhm) and an energy of ca. $3 \mathrm{~mJ}$. The light pulses were frequency doubled in an ADP-crystal with a 


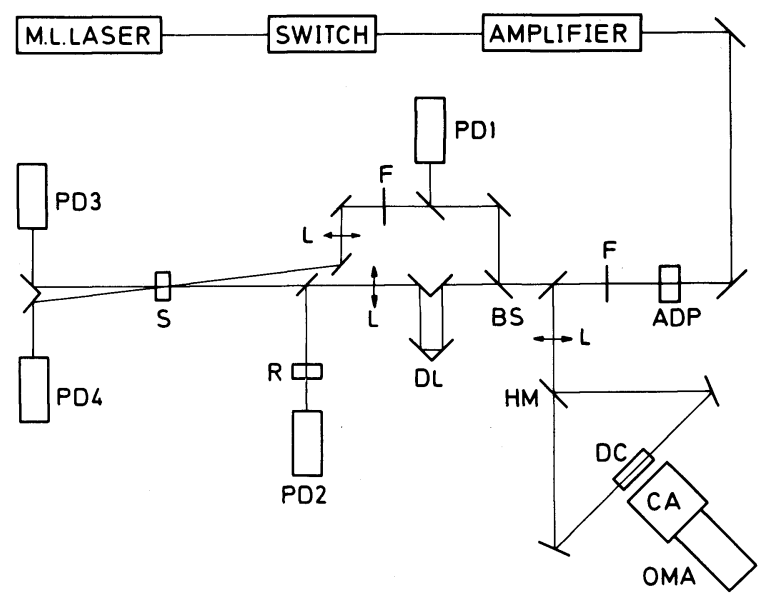

Fig. 1. Experimental set-up for the bleaching of dye solutions and the energy transmission measurements for pump and probe beam. ADP, crystal for second harmonic generation $(\lambda=5285 \AA) ; \mathrm{F}$, filter to block of fundamental frequency $(\lambda=1.06 \mu \mathrm{m}) ; \mathrm{L}$, lenses; HM, 50\% mirror; DC, two-photon fluorescing dye for pulse duration measurement; DA, camera; OMA, optical multi-channel analyser system; BS, beam splitter; DL, optical delay line; R, rutile crystal for intensity determination; PD1, PD2, PD3 and PD4 photodetectors; S, sample.

conversion efficiency of $\approx 20 \%$. The fundamental light at $\lambda=1.06 \mu \mathrm{m}$ was eliminated by a filter $\mathrm{F}$. The duration of the green light pulses $(\lambda=5285 \AA)$ was measured by a two-photon fluorescence system. 9,10-diphenyl-anthracene dissolved in cyclohexane $\left(10^{-3} \mathrm{M} / \ell\right)$ was used as two-photon absorber. A colliding beam arrangement was employed and the fluorescence light was imaged onto the vidicon of an optical multi-channel analyser (OMA). Pulse durations in the range between 3 ps and 5 ps were measured with an accuracy of 0.4 ps.

In fig. 1 the experimental arrangement is shown for the simultaneous measurement of the energy transmission of the strong pump pulse and the weak probe pulse. The probe pulse is generated from the main pump pulse with the help of the beam splitter BS. The delay line DL allows time-resolved transmission measurements of the probe pulse. The energy of the probe pulse was kept smaller than the pump beam by a factor of 32. The peak intensity of the pump pulse was determined by a two-photon absorption measurement using a rutile crystal $R$ [9]. The input energy and the transmitted energy were measured with fast photodetectors. Data are reported here on the mole- cules rhodamine $6 \mathrm{G}$ and rhodamine $\mathrm{B}$ measured in solutions of water, ethanol, ethylene glycol and glycerol. Most data were collected at room temperature. Measurements of rhodamine 6G dissolved in glassy ethanol were carried out at a temperature of $T=80$ $\mathrm{K}$.

(A) Energy transmission measurements of the intense picosecond light pulses were carried out in an intensity range between $10^{8} \mathrm{~W} / \mathrm{cm}^{2}$ and $10^{10} \mathrm{~W} / \mathrm{cm}^{2}$. Liquid cells of $1 \mathrm{~cm}$ length and dye concentrations of $10^{-5} \mathrm{M} / \ell$ were used in the experiments. At this low concentration dimerization of dye molecules is negligible [10]. The input pulse duration $t_{\mathrm{p}}$, the input peak intensity $I_{0 \mathrm{~L}}$ and the energy transmission $T_{\mathrm{L}}$ were measured simultaneously for each shot.

As an example, we present experimental data of rhodamine $6 \mathrm{G}$ dissolved in ethanol in fig. 2 . The open circles represent averages over five laser shots. Single pulses of duration $t_{\mathrm{p}}=3.5 \pm 0.5 \mathrm{ps}$ were chosen for the data points. It is readily seen from the figure that the transmission of the sample increases strongly from a low intensity value of $T_{0}=0.08$ to $T_{\mathrm{L}}=0.57$ for input peak intensities of $I_{0 \mathrm{~L}}=6 \times 10^{9} \mathrm{~W} / \mathrm{cm}^{2}$. The two curves in fig. 2 are calculated for population life-

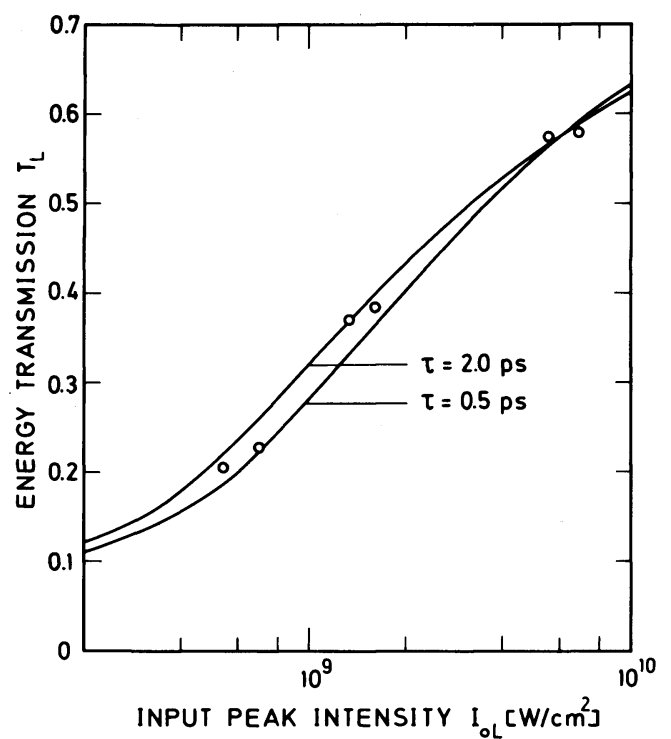

Fig. 2. Energy transmission of intense picosecond light pulses through rhodamine $6 \mathrm{G}$ dissolved in ethanol. Concentration $10^{-5} \mathrm{M} / \mathrm{\ell}$; sample length $1 \mathrm{~cm}$; absorption cross section $\sigma_{12}=$ $1.25 \times 10^{-15} \mathrm{~cm}^{2}$; open circles correspond to experimental data.The curves are calculated for $\tau=0.5, \tau=2.0 \mathrm{ps}$ and $t_{\mathrm{p}}=3.5 \mathrm{ps}$. The $\sigma$-values are $2 \times 10^{-16} \mathrm{~cm}^{2}$ and $1.6 \times 10^{-16}$ $\mathrm{cm}^{2}$ for $\tau=2 \mathrm{ps}$ and $0.5 \mathrm{ps}$, respectively. 
times of $\tau=0.5 \mathrm{ps}$ and $\tau=2.0 \mathrm{ps}$ using an energy level model which will be discussed below. The theoretical curves account well for our experimental data suggesting a time constant of $\tau \approx 1 \mathrm{ps}$.

(B) The transmission of a subsequent weak probe pulse allows an independent determination of the relaxation time $\tau$ of the initially excited energy states. For a fixed intensity of the pump pulse, the energy transmission $T_{\|}$of a parallel polarized interrogating pulse is determined. The ratio of the energy transmissions $R=T_{\|} / T_{\mathrm{L}}$ changes as a function of delay time $t_{\mathrm{D}}$ depending upon the time constant $\tau$. For $\tau \gtrsim t_{\mathrm{p}} / 4$ the time dependence of the $R\left(t_{\mathrm{D}}\right)$ curves allows an accurate determination of the relaxation time. For $\tau \lesssim t_{\mathrm{p}} / 4$ the shape of the $R\left(t_{\mathrm{D}}\right)$ curves becomes similar and the relaxation time has to be determined from the absolute values of $R\left(t_{\mathrm{D}}\right)$.

We have measured the energy transmission ratio $R$ as a function of delay time $t_{\mathrm{D}}$ for various solutions and compared it with calculated curves as indicated below. Concentrations of $10^{-4} \mathrm{M} / \ell$ and sample lengths of 0.2 $\mathrm{cm}$ (rhodamine 6G) and $0.5 \mathrm{~cm}$ (rhodamine B) were used. In fig. 3 we present experimental data (open circles) for rhodamine $6 \mathrm{G}$ in ethanol. Fig. 3 shows quite vividly the rapid rise of the probe transmission around $t_{\mathrm{D}} \approx 0$ and the decay of the transmission values for longer delays $t_{\mathrm{D}}$ between the pump and the probe pulse. For delay times $t_{\mathrm{D}}$ long compared to $\tau$ the decay of the curves is determined by the orientational time constant $\tau_{\text {or }}$ which is known from the literature [11]. Our experimental points of $R\left(t_{\mathrm{D}}\right)$ are well accounted for by a theoretical curve with relaxation parameter $\tau \approx 0.7 \mathrm{ps}$.

Similar results obtained for rhodamine $6 \mathrm{G}$ in water, ethylene glycol and glycerol and for rhodamine B in water, ethanol and glycerol at room temperature (see table 1) and for rhodamine $6 \mathrm{G}$ in ethanol at $80 \mathrm{~K}$.

We turn now to a quantitative discussion of our observations. A five-level system appears to be appropriate for a dynamic description of the experimental situation. Fig. 4 shows schematically the considered level system of the rhodamine molecules (right) together with the measured absorption spectrum (left) of rhodamine $6 \mathrm{G}$ in ethanol. The calculated model has the following features:

(i) Prior to optical excitation thermal equilibrium exists in the $\mathrm{S}_{0}$ ground state position (1) with energy spread $\Delta E \approx k T \approx 200 \mathrm{~cm}^{-1}$ at $T \approx 300 \mathrm{~K}$.

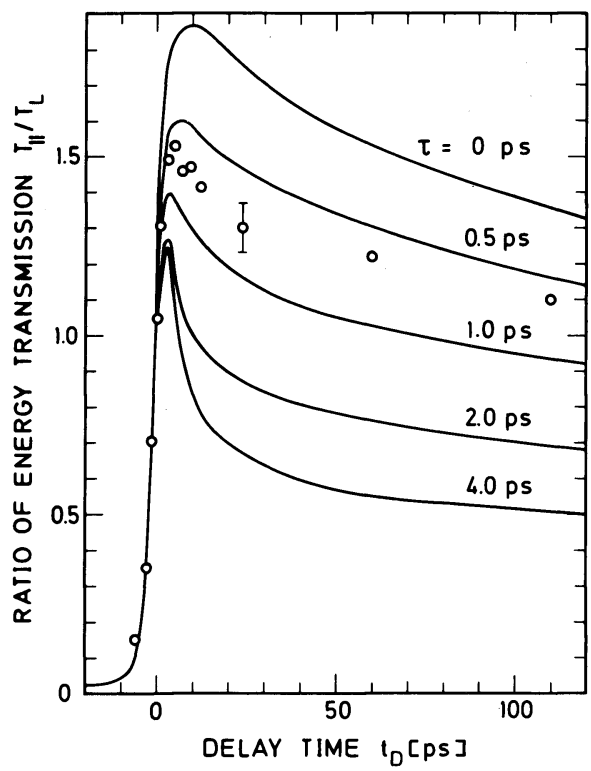

Fig. 3. Time dependent energy transmission of a probe pulse through a rhodamine $6 \mathrm{G}$ solution in ethanol. Dye concentration $10^{-4} \mathrm{M} / \mathrm{l}$, sample length $0.2 \mathrm{~cm}, T=300 \mathrm{~K}$. The probe pulse is polarized parallel to the pump pulse. The curves are calculated for various decay times $\tau$. The circles represent experimental data. The input pulse parameters are, $t_{\mathrm{p}}=3.7 \mathrm{ps}$, peak intensities $I_{0 \mathrm{~L}}=3 \times 10^{9} \mathrm{~W} / \mathrm{cm}^{2}, I_{0 \mathrm{P}}=9 \times 10^{7} \mathrm{~W} / \mathrm{cm}^{2}$. The orientational relaxation time of $\tau_{\text {or }}=270$ ps was taken from the literature [11].

(ii) A linear polarized picosecond light pulse of frequency $\tilde{\nu}_{\mathrm{L}}=18910 \mathrm{~cm}^{-1}$ excites molecules to a nonequilibrium position (2) in the $S_{1}$ state (FranckCondon state with energy spread equal to that of the ground state). A constant absorption cross section $\sigma_{12}$ of the thermally populated ground state levels is assumed since transition probabilities to the respective $\mathrm{S}_{1}$ states are expected to have nearly equal FranckCondon factors.

(iii) The energy states (2) are emptied by vibrational relaxation and reorientation to a temporary equilibrium position (3) in the $S_{1}$ state. The position (3) is situated approximately $600 \mathrm{~cm}^{-1}$ and $1300 \mathrm{~cm}^{-1}$ below the position (2) in rhodamine $6 \mathrm{G}$ and rhodamine B solutions, respectively.

(iv) Most molecules in the $S_{1}$ band return to the ground state by fluorescence emission. The fluorescence lifetimes are in the nanosecond range with $\tau_{\mathrm{F}}$ $\approx 4.5 \mathrm{~ns}$ and $\tau_{\mathrm{F}} \approx 2.3 \mathrm{~ns}$ for rhodamine $6 \mathrm{G}$ [12] and rhodamine B [13], respectively. Complications due to 
Table 1

\begin{tabular}{|c|c|c|c|c|c|c|c|}
\hline \multirow[t]{2}{*}{ Solution } & \multirow{2}{*}{$\begin{array}{l}10^{16} \sigma_{12} \\
\left(\mathrm{~cm}^{2}\right)\end{array}$} & \multirow{2}{*}{$\begin{array}{l}10^{16} \sigma_{15} \\
\left(\mathrm{~cm}^{2}\right)\end{array}$} & \multirow{2}{*}{$\begin{array}{l}10^{16} \sigma_{\mathrm{ex}} \\
\left(\mathrm{cm}^{2}\right)\end{array}$} & \multirow{2}{*}{$\begin{array}{l}\eta \\
\text { (cP) }\end{array}$} & \multirow{2}{*}{$\begin{array}{l}\tau_{\text {or }} \\
\text { (ps) }\end{array}$} & \multicolumn{2}{|l|}{$\tau$} \\
\hline & & & & & & $\begin{array}{l}\text { bleaching } \\
\text { (ps) }\end{array}$ & $\begin{array}{l}\text { probing } \\
\text { (ps) }\end{array}$ \\
\hline $\begin{array}{l}\text { rhodamine } 6 \mathrm{G} \text { in } \\
\text { water } \\
\text { ethanol } \\
\text { ethylene glycol } \\
\text { glycerol }\end{array}$ & $\begin{array}{l}11.1 \\
12.5 \\
11.4 \\
10.4\end{array}$ & $\begin{array}{l}1.75 \\
1.7 \\
2.3 \\
1.55\end{array}$ & $\begin{array}{l}1.8 \pm 0.2 \\
1.8 \pm 0.2 \\
3.0 \pm 0.3 \\
1.4 \pm 0.2\end{array}$ & $\begin{array}{l}1.0 \\
1.19 \\
19.9 \\
1470\end{array}$ & $\begin{array}{r}230 \\
270 \\
2000 \\
>2000\end{array}$ & $\begin{array}{l}1.2 \pm 0.8 \\
1.2 \pm 0.8 \\
0.8 \pm 0.7\end{array}$ & $\begin{array}{l}0.7 \pm 0.3 \\
0.7 \pm 0.2 \\
0.8 \pm 0.2 \\
0.8 \pm 0.2\end{array}$ \\
\hline $\begin{array}{l}\text { rhodamine B in } \\
\text { water } \\
\text { ethanol } \\
\text { glycerol }\end{array}$ & $\begin{array}{l}4.4 \\
5.6 \\
3.2\end{array}$ & $\begin{array}{l}2.5 \\
2.7 \\
1.85\end{array}$ & $\begin{array}{l}1.7 \pm 0.2 \\
1.6 \pm 0.2 \\
1.3 \pm 0.2\end{array}$ & $\begin{array}{c}1.0 \\
1.19 \\
1470\end{array}$ & $\begin{array}{r}230 \\
270 \\
>2000\end{array}$ & $\begin{array}{l}1.2 \pm 1 \\
1.2 \pm 1 \\
1.2 \pm 1\end{array}$ & $\begin{array}{l}0.9 \pm 0.3 \\
0.9 \pm 0.3 \\
0.8 \pm 0.5\end{array}$ \\
\hline
\end{tabular}
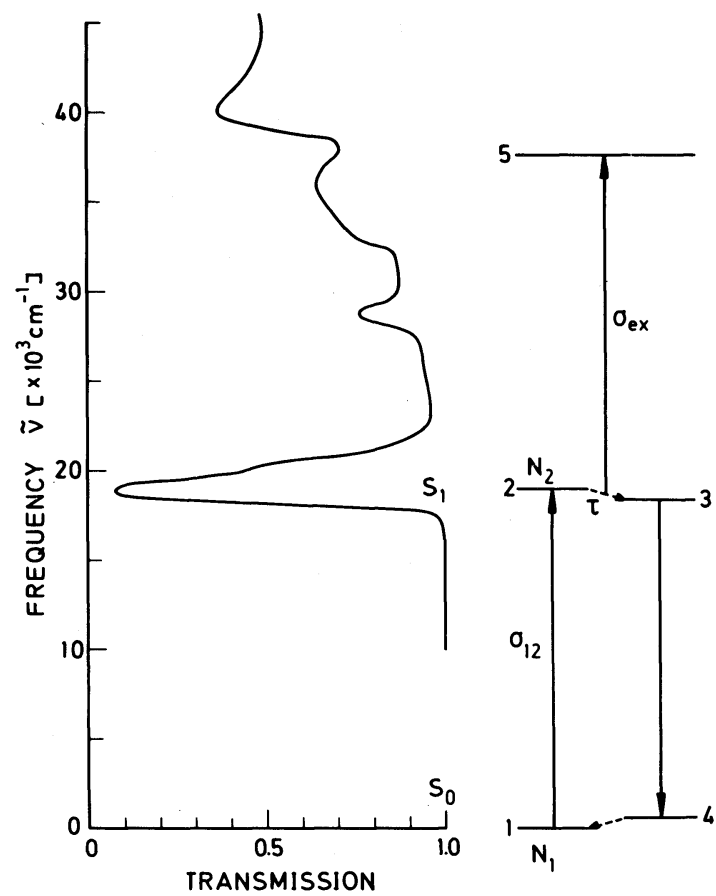

Fig. 4. Absorption spectrum of rhodamine $6 \mathrm{G}$ in ethanol $\left(10^{-5} \mathrm{M} / \ell, 1 \mathrm{~cm}\right.$ cell length) and schematic five-level model for the transitions in rhodamine dyes.

super-fluorescence do not occur in our experiments as checked by measurements of the fluorescence decay times and the emission spectra of our dye solutions. Repopulation of the ground state by fluorescence is neglected.

(v) Molecules in the $S_{1}$ state absorb pump light of
$\tilde{\nu}_{\mathrm{L}}$ by excited state absorption and thereby populate higher singlet states (position 5). A substantial accumulation of population in these higher singlet states does not occur since the excited state absorption cross section $\sigma_{\mathrm{ex}}$ is small and the relaxation to position (3) is expected to be in the picosecond range. We assume a constant value of $\sigma_{\mathrm{ex}}$ for all molecules which are in the electronically excited state.

(vi) Transfer of molecules from the $S_{1}$ state to a triplet state is negligibly small [14].

(vii) Strong electric dipole transitions are responsible for the excitation of the molecules to higher lying electronic states. The dipole transition moments have fixed directions within the molecule. The complex dye molecules can be described classically as linear oscillators [15]. The strength of the light absorption depends upon the angle $\theta$ between dipole transition moment and polarization of the light beam. The absorption cross section is given by $* \sigma(\theta)=$ $\sigma \cos ^{2} \theta$. For the molecules investigated here the transition moments for ground state absorption $\sigma_{12}$ and excited state absorption $\sigma_{\mathrm{ex}}$ have the same direction as shown by fluorescence polarization measurements [16] . At the beginning of the absorption process the transition moments are oriented isotropically and the average

* An experimental check of this formula will be given in a following paper. Small deviations from this ideal linear oscillator model are found. An extended model with the ansatz $\sigma=\sigma_{\|} \cos ^{2} \theta+\sigma_{\perp} \sin ^{2} \theta$ leads to an increase of the $\tau$ values by approximately 0.2 ps and reduces the excited state absorption cross sections by approximately $5 \%$ compared to the values listed in table 1 . 
absorption cross section is $\sigma_{\mathrm{m}}=\sigma / 3$. Cross section $\sigma_{\mathrm{m}}$ is measured with conventional photometric techniques. The intense picosecond pump pulse excites preferentially those molecules with their dipole moments oriented under a small angle to the linearly polarized light field.

(viii) Rotational brownian motion requires times exceeding $10^{-10} \mathrm{~s}$ depending upon the solvents used in our experiments. As a result the reorientation of the dye molecules has no influence on the bleaching process by the strong picosecond light pulse but the transmission of the probe pulses is reduced for long delay times by rotational redistribution.

Rate equations are used for the interaction of the light pulses with the dye molecules. This classical approach is justified since the dephasing time $T_{2}$ is expecting to be considerably shorter than the laser pulse duration $\left(T_{2}<0.5 \mathrm{ps} ; t_{\mathrm{p}} \approx 4 \mathrm{ps}\right)$. The following equations describe the bleaching of a strong pump pulse and the transmission behavior of a weak delayed probe beam of parallel polarization.

$$
\begin{aligned}
& \frac{\partial I_{\mathrm{L}}}{\partial z}+\frac{n}{c} \frac{\partial I_{\mathrm{L}}}{\partial t}=-I_{\mathrm{L}} \int_{0}^{\pi / 2} \sin \theta \cos ^{2} \theta \\
& \quad \times\left\{\sigma_{12}\left[N_{1}(\theta)-N_{2}(\theta)\right]+\sigma_{\mathrm{ex}}\left[N-N_{1}(\theta)\right]\right\} \mathrm{d} \theta \\
& \frac{\partial I_{\mathrm{P}}}{\partial z}+\frac{n}{c} \frac{\partial I_{\mathrm{P}}}{\partial t}=-I_{\mathrm{P}} \int_{0}^{\pi / 2} \sin \theta \cos ^{2} \theta \\
& \quad \times\left\{\sigma_{12}\left[N_{1}(\theta)-N_{2}(\theta)\right]+\sigma_{\mathrm{ex}}\left[N-N_{1}(\theta)\right]\right\} \mathrm{d} \theta
\end{aligned}
$$

$$
\begin{gathered}
\frac{\partial N_{1}(\theta)}{\partial t}=-\frac{1}{h \nu_{\mathrm{L}}} \sigma_{12} \cos ^{2} \theta\left(I_{\mathrm{L}}+I_{\mathrm{P}}\right)\left[N_{1}(\theta)-N_{2}(\theta)\right] \\
-\frac{N_{1}(\theta)-\int_{0}^{\pi / 2} \sin \rho N_{1}(\rho) \mathrm{d} \rho}{\tau_{\text {or }}}
\end{gathered}
$$

$$
\begin{aligned}
& \frac{\partial N_{2}(\theta)}{\partial t}=-\frac{1}{h \nu_{\mathrm{L}}} \cos ^{2} \theta\left\{\sigma_{12}\left(I_{\mathrm{L}}+I_{\mathrm{P}}\right)\left[N_{1}(\theta)-N_{2}(\theta)\right]\right. \\
& \left.-\sigma_{\mathrm{ex}}\left(I_{\mathrm{L}}+I_{\mathrm{P}}\right) N_{2}(\theta)\right\}-N_{2}(\theta) / \tau \\
& =\frac{N_{2}(\theta)-\int_{0}^{\pi / 2} \sin \rho N_{2}(\rho) \mathrm{d} \rho}{\tau_{\text {or }}}
\end{aligned}
$$

The initial conditions are

$$
I_{\mathrm{L}}(t, r, z=0)=I_{0 \mathrm{~L}} \exp \left[-\left(t / t_{0}\right)^{2}-\left(r / r_{0}\right)^{2}\right] \text {, }
$$

$$
\begin{aligned}
& I_{\mathrm{P}}(t, r, z=0) \\
& \left.\quad=I_{0 \mathrm{P}} \exp \left\{-\left[t-t_{\mathrm{D}}\right) / t_{0}\right]^{2}-\left(r / r_{0}\right)^{2}\right\}, \\
& N_{1}(t-n z / c=-\infty, r, z, \theta)=N, \\
& N_{2}(t-n z / c=-\infty, r, z, \theta)=0 .
\end{aligned}
$$

$I_{0 \mathrm{~L}}$ and $I_{0 \mathrm{P}}$ are the peak intensities of the incident picosecond pump and probe pulse, respectively. Gaussian pulse shapes are assumed. $2 t_{0}$ and $r_{0}$ are the $1 / \mathrm{e}$ values of the pulse duration and the beam radius of both pulses. $\nu_{\mathrm{L}}$ is the frequency of the laser pulses (both pulses have the same frequency), $n$ denotes the refractive index. $N=\int_{0}^{\pi / 2} N(\theta) \sin \theta \mathrm{d} \theta$ is the total number density of dye molecules in the solution. $N_{1}(\theta)$ and $N_{2}(\theta)$ are the number densities of molecules in the ground state (1) and the Franck-Condon state (2). The rotational redistribution of the molecules is taken into account phenomenologically by the last terms in eqs. (3) and (4). The equations are simplified by the transformation $z^{\prime}=z$ and $t^{\prime}=t-$ $n z / c$. The resulting system is solved numerically and the transmitted intensities $I_{\mathrm{L}}\left(t^{\prime}, r, z^{\prime}=l\right)$ and $I_{\mathrm{P}}\left(t^{\prime}, r, z^{\prime}=l\right)$ are calculated. The energy transmissions for the incident pump pulse, $T_{\mathrm{L}}$, and for the probe pulse, $T_{\|}$, are $(i=\mathrm{L}, \|)$

$$
T_{i}=\frac{\int_{0}^{\infty} 2 \pi r \int_{-\infty}^{\infty} I_{i}\left(t^{\prime}, r, l\right) \mathrm{d} t^{\prime} \mathrm{d} r}{\int_{0}^{\infty} 2 \pi r \int_{-\infty}^{\infty} I_{i}\left(t^{\prime}, r, 0\right) \mathrm{d} t^{\prime} \mathrm{dr}} .
$$

The energy transmissions $T_{\mathrm{L}}$ and $T_{\|}$are determined by the material parameters $N, \sigma_{12}, \sigma_{\text {ex }}, \tau_{\text {or }}, \tau$, the pulse parameters $I_{0 \mathrm{~L}}, I_{0 \mathrm{P}}, t_{0}$ and the delay time $t_{\mathrm{D}}$. $N$ is determined by the chosen dye concentration. $\sigma_{12}=3 \sigma_{\mathrm{m}}$ is obtained by absorption measurements with a spectral photometer. $\tau_{\text {or }}$ is known from the literature [11]. For each laser shot we measured the peak intensities $I_{0 \mathrm{~L}}, I_{0 \mathrm{P}}$ and the pulse duration $t_{\mathrm{p}}=2(\ln 2)^{1 / 2} t_{0}(\mathrm{fwhm})$ together with the energy transmission $T_{\mathrm{L}}$ and $T_{\|}$.

To (A). The nonlinear transmission of an intense picosecond laser pulse is calculated from eqs. (1)(8) with the simplification of $I_{0 \mathrm{P}}=0$ (no probe pulse). Two calculated curves for the population lifetimes $\dot{\tau}=0.5$ and 2 ps are dipicted in fig. 2. The excited state absorption cross section $\sigma_{\text {ex }}$ was chosen in such a way that the curves fit the experimental data at the highest intensity value of $I_{0 \mathrm{~L}}=6 \times 10^{9} \mathrm{~W} / \mathrm{cm}^{2}$. In 
table 1 , the experimentally determined values of $\sigma_{\text {ex }}$ are compared with the absorption cross sections $\sigma_{15}=3 \sigma_{15 \mathrm{~m}}$ which start from the ground state (1) $\left(\widetilde{v}=37800 \mathrm{~cm}^{-1}\right)$. In rhodamine $6 \mathrm{G}$ the two cross sections are practically the same, in rhodamine $\mathrm{B}$, the values of $\sigma_{15}$ appear to be somewhat larger.

The comparison of the experimental points with the calculated curves allows to estimate the time constant $\tau$ to lie between 0.5 ps and 2 ps. The numbers obtained for various solutions are listed in table 1 together with estimated uncertainties.

To (B). The energy transmission of a probe pulse was calculated as a function of delay time $t_{\mathrm{D}}$ for different population lifetimes $\tau$. The five curves in fig. 3 show strongly varying shapes for $\tau$-values between 0 ps and 4 ps. The comparison between the calculated curves and the experimental points allows us to determine the relaxation time $\tau$ with an uncertainty of $\pm 0.2 \mathrm{ps}$. This accuracy is considerably better than found in the simple pulse transmission of point $\mathrm{A}$. The obtained times are summarized in table 1 for $T=300 \mathrm{~K}$. Values of $\tau$ between $0.5 \mathrm{ps}$ and $1 \mathrm{ps}$ are found for all investigated solutions of rhodamine $6 \mathrm{G}$ and rhodamine $\mathrm{B}$, practically independent of the viscosity of the solvent. We note that even in the solid solution at $T=80 \mathrm{~K}$ the short lifetime of $\tau=0.9 \pm 0.2 \mathrm{ps}$ is observed.

The measured $\tau$-values determine the lifetime of the molecules in the initially excited states (2). The decay of the molecules from the resonant Franck-Condon states by reorientation of the solvent molecules is ruled out on account of the viscosity data. Reorientation is too slow in highly viscous solutions and in particular in the glassy sample at $80 \mathrm{~K}$. Two physical processes contribute to the observed rapid changes of optical transmission. (1) Energy transfer to neighboring vibrational states with smaller absorption cross sections and (2) vibrational decay to lower lying vibrational levels within the $S_{1}$ state. It appears at the present time that both processes (1) and (2) contribute to our observations.

Our numbers of the vibrational relaxation times differ somewhat from previous reports $[1-7]$. This result is expected since the relaxation values are deduced from different experiments. In this letter the transient population of certain vibrational energy levels is studied while previous investigations determined the delay between excitation and the appearance of gain or measured the delayed appearance of fluorescence spectra.

The authors thank Dr. A. Laubereau for valuable discussions.

\section{References}

[1] D. Ricard, W.H. Lowdermilk and J. Ducuing, Chem. Phys. Letters 16 (1972) 617.

[2] D. Ricard, J. Chem. Phys. 63 (1975) 3841.

[3] E.P. Ippen and C.V. Shank, Opt. Commun. 18 (1976) 27.

[4] M.M. Malley and G. Mourou, Opt. Commun. 10 (1974) 323.

[5] G. Mourou and M.M. Malley, Chem. Phys. Letters 32 (1975) 476.

[6] A.N. Rubinov, M.C. Richardson and A.J. Alcock, Soviet J. Quantum Electron. 5 (1976) 910.

[7] P.M. Rentzepis, M.R. Topp, R.P. Jones and J. Jortner, Phys. Rev. Letters 25 (1970) 1742.

[8] A. Laubereau and W. Kaiser, Opto-Electronics 6 (1974) 1.

[9] A. Penzkofer and W. Falkenstein, Opt. Commun. 17 (1976) 1.

[10] Th. Förster and E. König, Z. Elektrochem. 61 (1957) 344.

[11] T.J. Chuang and K.B. Eisenthal, Chem. Phys. Letters 11 (1971) 368;

H.E. Lessing, A. von Jena and M. Reichert, Chem. Phys. Letters 36 (1975) 517.

[12] K.H. Drexhage, in: Topics of applied physics, Vol. 1, ed. F.P. Schäfer (Springer, Berlin, 1974) p. 149.

[13] H.R. Stadelmann, J. Luminescence 3 (1970) 143.

[14] D.N. Dempster, T. Morrow and M.F. Quinn, J. Photochem. 2 (1973) 343;

V.E. Korobov and A.K. Chibisov, Opt. Spectry. 38 (1975) 706.

[15] P.P. Feofilov, The physical basis of polarized emission (Consultants Bureau, New York, 1961);

F. Dörr and M. Held, Angew. Chem. 72 (1960) 287.

[16] H. Jakobi and H. Kuhn, Z. Elektrochem. 66 (1962) 46. 